\title{
Maxillary premolars extraction or molar distalization with or without TAD's: cephalometric evaluation of soft tissue changes in Class II treatment
}

\author{
1 Private practice in Bolzano, Deptartment of Orthodontics, University of Ferrara, Ferrara, Italy \\ 2 Private practice in Milan, Deptartment of Medicine and Surgery, University of Insubria, Varese, Italy \\ 3 Deptartment of Medicine and Surgery, University of Insubria, Varese, Italy \\ 4 Private practice in Vicenza, Deptartment of Medicine and Surgery, University of Insubria, Varese, Italy \\ 5 United Campus of Malta, Malta \\ 6 Deptartment of Medicine and Surgery, University of Insubria, Varese, Italy
}

Lorenz Moser $^{1}$; Enrica Di Lorenzo ${ }^{2}$; Marco Serafin ${ }^{3}$; Giuliano Maino ${ }^{4}$; Ute Schneider-Moser ${ }^{1}$; Mauro Cozzani ${ }^{5}$; Alberto Caprioglio ${ }^{6}$

\begin{abstract}
Introduction: Problem-solving in Class II malocclusion treatment performed with premolars extractions or distalizing techniques in relation to the profile modification.

The aim: To cephalometrically compare soft tissue changes produced either by maxillary premolar extraction, tooth-borne Pendulum appliance or bone-borne MGBM appliance.

Material and methods: Both pre- (T1) and post-treatment (T2) lateral cephalograms of 89 skeletal Class II patients (36 M, $53 \mathrm{~F})$, treated during pubertal growth spurt, were retrospectively selected. Three groups were formed based on the therapy performed: 30 patients had been treated with maxillary first premolars extraction (U4), 31 patients with a conventional tooth-borne distalizing with Pendulum appliance (PA), and 28 patients with a skeletally anchored distalizing appliance (MGBM). Soft tissue was analyzed comparing upper (UL) and lower (LL) lip's points with regard to True Vertical Line (TVL) and Esthetic plane (E-plane). Skeletal and dental values have been recorded in order to cephalometrically compare $\Delta \mathrm{T} 2-\mathrm{T} 1$ changes among groups and to correlate dental and skeletal changes to profile modifications. One-way ANOVA was employed to compare groups at T1. Paired sample t-tests were employed to assess significant intra- and intergroup differences between T2 and T1. Significance level was set at 0.05 .

Results: UL and LL showed a slight but not significant retrusion relative to TVL in all three groups. UL and LL distances to E-plane were not statistically significant among U4, PA, and MGBM groups. Independently of the treatment, UL was tangent to TVL in all groups. No statistically significant differences have been shown in skeletal records. Significant differences were recorded in Overjet among U4 than PA and MGBM groups.
\end{abstract}

Conclusions: Class II malocclusion treatment with maxillary first premolar extraction, conventional or skeletal distalization did not significantly affect the profile producing similar changes in the soft tissue.

Moser L, Di Lorenzo E, Serafin M, Maino G, Schneider-Moser U, Cozzani M, Caprioglio A. Maxillary premolars extraction or molar distalization with or without TAD's: cephalometric evaluation of soft tissue changes in Class II treatment. South Eur J Orthod Dentofac Res. 2020;7(1):4-11.

\section{INTRODUCTION}

Class II malocclusion is one of the most frequent problems that orthodontists diagnose in their daily practice. Due to that,

Corresponding Author:

Marco Serafin,

Via IV Novembre, 2 - 21017 - Samarate, Italy

e-mail: marco.serafin.1990@gmail.com treatment of Class II malocclusion is considered one of the most investigated and controversial issues in contemporary orthodontic practice, as it may affect facial harmony, depending on dental overjet and its interaction with soft tissue, ${ }^{1}$ especially in changes in the position and contour of lips. ${ }^{2}$ Moreover, improvement in facial esthetics has become an aspect of utmost importance in contemporary society, as one of the major motivations for seeking orthodontic treatment. In fact, one of the goals of the Class II treatment is to gain a straighter profile from a convex one. ${ }^{3}$ 
Class II malocclusions are treated with functional orthopedic appliances, ${ }^{4}$ extraoral ${ }^{5}$ and intraoral ${ }^{6}$ appliances for maxillary tooth distalization, dental extractions ${ }^{7}$ or surgical-orthodontic procedures. ${ }^{8,9}$ If there are no significant skeletal discrepancy and tooth crowding, Class II can be successfully corrected by molar distalization or premolar extractions.

Camouflage treatment can be performed by the extraction of first upper premolars, especially in crowded arch; the maximum anchorage is then required to retract the anterior teeth maximally. ${ }^{7}$ The esthetic impact of soft tissue profile might play a major role in deciding on premolar extraction or nonextraction treatment, ${ }^{10}$ along with crowing, ${ }^{11}$ particularly in borderline patients who can approach to distalizing techniques.

Pendulum appliance is one of the tools that has been more investigated since it has been developed in $1992 .{ }^{12}$ Pendulum is used in Class II malocclusions, especially those related with maxillary prognathism. ${ }^{13}$ However, undesired premolar anchorage loss can be expected, which can lead to a prolonged treatment time and a subsequent need for cooperation during retraction of premolar, canine and incisor after distalization.

In order to overcome the undesired side effects of dental-borne appliances, involving skeletal anchorage solutions, the noncompliance MGBM system was introduced in $2006 .{ }^{14}$ In this technique, the anchorage is provided by a transpalatal bar, which is bonded to the maxillary first premolars and to which two palatal miniscrews are connected; after molar distalization, two buccal miniscrews are inserted to provide absolute anchorage for anterior teeth distalization finalizing in Class I canine relation and reducing overjet. ${ }^{15}$

Maxillary premolars extractions for Class II correction purposes have been widely discussed in literature, because the effect of upper incisor retraction may compromise the patient's profile. ${ }^{16}$ However, scientific evidence suggests that extractions, when indicated, does neither harm the profile or facial attractiveness. Research on dental and skeletal cephalometric effects and treatment outcomes between distalizing and extraction treatments has already been published, ${ }^{17,18}$ but the effects on soft tissue outcomes are still controversial.

The purpose of the present investigation was to test the following null hypothesis that there is no difference in soft tissue profile changes of Class II malocclusion treated with either maxillary first premolars extractions, tooth-borne molar distalization with Pendulum appliance or skeletal-borne molar distalization with MGBM appliance.

\section{MATERIAL AND METHODS}

The initial sample of the present retrospective study consisted of 111 patients selected from three dental offices of Board-Certified orthodontists. Signed informed consent for releasing diagnostic records for scientific purposes was obtained from the parents of the patients, prior to entry into the orthodontic treatment. Sample size was calculated on the measurements of two patients per group selecting as main outcome the distance between the true vertical line and upper central incisor (//TVL-VeU1) changes before and after treatment (mean difference $=-1.05$; standard deviation $=1,93$ ). Sample size of at least 25 subjects per group (total sample size $=75$ ) was necessary to detect a power of $0.8(\beta=0.20)$ with $\alpha$ set at 0.05 . Considering the retrospective design of the study, a certain amount of dropouts percentage should be provided, then a greater number of patients was selected. Among all patients only who satisfied inclusion and exclusion criteria were selected for the final group.

Sample selection and exclusion criteria

- Mild or moderate skeletal Class II $\left(\mathrm{ANB} \leq 6^{\circ}\right)$ with at least dental end-to-end Class II molar relationships;

- Mandibular inclination ( $\left(\mathrm{N}^{\wedge} \mathrm{GoGn}\right)$ less than $37^{\circ}$;

- Aged between 11 to 14 years old;

- No previous orthodontic treatment, no previous serial extraction treatment;

- Permanent dentition (except third molars);

- Absence of any dental anomalies in number, size and form;

- Absence of posterior crossbite;

- Mandibular arch with minimal crowding $(<2 \mathrm{~mm})$;

- Good quality radiographs with adequate landmark visualization, obtained at baseline (T1) and after fixed appliance treatment (T2).

From the initial sample of 111 patients, the records of 22 patients were excluded, according to defined criteria. The final sample consisted of 89 patients divided into 3 groups as summarized in Table 1.

Table 1. Sample selection and exclusion criteria.

\begin{tabular}{|c|c|c|c|}
\hline \multirow[t]{2}{*}{ Patient sample } & \multicolumn{3}{|c|}{111} \\
\hline & $\begin{array}{l}\text { U4 } \\
41\end{array}$ & $\begin{array}{l}\text { PA } \\
36\end{array}$ & $\underset{34}{\text { MGMB }}$ \\
\hline \multicolumn{4}{|l|}{ Primary exclusion criteria: } \\
\hline 1. Poor film quality & 3 & 3 & 2 \\
\hline 2. Incomplete records & 1 & 0 & 1 \\
\hline \multicolumn{4}{|l|}{ Secondary exclusion criteria: } \\
\hline 1. Mandibular inclination $\left(\mathrm{SN}^{\wedge} \mathrm{Go}-\mathrm{Gn}>37\right)$ & 2 & 2 & 2 \\
\hline 2. Non-Class II malocclusion & 2 & 0 & 1 \\
\hline 3. Use of other molar distalization methods & 1 & 0 & 0 \\
\hline \multirow[t]{2}{*}{ 4. Extraction upper 5 due to cavity } & 2 & 0 & 0 \\
\hline & 30 & 31 & 28 \\
\hline Final sample & & 89 & \\
\hline
\end{tabular}

U4: upper first premolar extraction group; PA: Pendulum appliance group; MGBM: skeletal appliance group.

\section{Clinical management}

- UP4 group. The maxillary first premolars were extracted before the onset of orthodontic therapy. Subsequently, a preadjusted fixed orthodontic straight-wire appliance (Roth prescription, $0.022 \times 0.028$-in slot) was bonded and leveling and aligning started with an initial 0.014-in or 0.016-in NiTi wire, followed by a $0.017 \times 0.025$-in NiTi wire. Space closure was performed with a $0.019 \times 0.025$-in SS wire and sliding mechanics (elastic o-chains) in conjunction with Class II elastics. 
- PA group. All patients underwent maxillary molar distalization therapy with a Pendulum appliance ${ }^{11}$ with uprighting bends to prevent excessive molar tipping. ${ }^{19,20}$ The appliance was left in situ until a super Class I molar relationship was achieved. After removal of the Pendulum appliance, a Nance button was placed and preadjusted fixed orthodontic appliances (Roth prescription, $0.022 \times 0.028$-in slot) were used to retract the premolars, canines and incisors using sliding mechanics and intermaxillary Class II elastics.

- MGBM group. Twenty-eight patients were treated with MGBM-system. ${ }^{14,21}$ The first phase of Class II treatment was performed by miniscrew-borne distalization of the maxillary molars into an overcorrected Class I relationship. After distalization, the palatal miniscrews were removed and placed buccally (between the first molar and second premolar) for anchorage preservation. To respect the indications provided by the inventors of this technique, a preadjusted fixed bidimensional straight-wire appliance $(0.018 \times 0.025$ in slot size on incisors and canine and $0.022 \times 0.028$-in slot size on cuspid premolars and molars) was used to retract the premolars, canines and incisors with closed coil springs and intermaxillary elastics.

\section{Cephalometric analysis}

In all three groups the lateral cephalograms at $\mathrm{T} 1$ and $\mathrm{T} 2$ were standardized at the same magnification factor ( $6 \%$ enlargement). All of the cephalometric radiographs were hand-traced on acetate paper by one investigator with verification of anatomic outlines and landmark placement by a second investigator. In case of disagreement, the structures in question were retraced to the satisfaction of both operators. In the case of divergent bilateral structures, the two traced points were averaged to one single point. After the identification of 26 landmarks (Figure 1) and 15 reference planes (Figure 2), a comprehensive hard and soft tissue cephalometric analysis with 9 angular and 11 linear measurements was performed for each patient at T1 and T2. The superimpositions were performed using Bjork's structural method. $\mathrm{SN}^{\wedge} \mathrm{GoGn}$ was used as skeletal parameter for vertical facial dimension. TVL and E-plane were used as references to assess sagittal soft tissue changes together with the evaluation of Naso-labial Angle (NLA).

\section{Statistical Analysis}

All statistical analyses were performed with the SPSS software, version 22.0 (SPSS ${ }^{\odot}$ Inc., Chicago, IL, USA). Parametrical methods were used after assessing normal distribution with the Shapiro-Wilk test and equality of variances with Levene test. Descriptive statistics included calculation of means and standard deviations. An analysis of variance (ANOVA) was utilized to compare groups at T1. Paired sample t-test was employed for assessing any significant difference of each measurement between time points within each group. Null hypothesis was rejected when a p-value scored below 0.05 . ANOVA was used to compare differences between $\mathrm{T} 1$ and $\mathrm{T} 2$ of each measurement

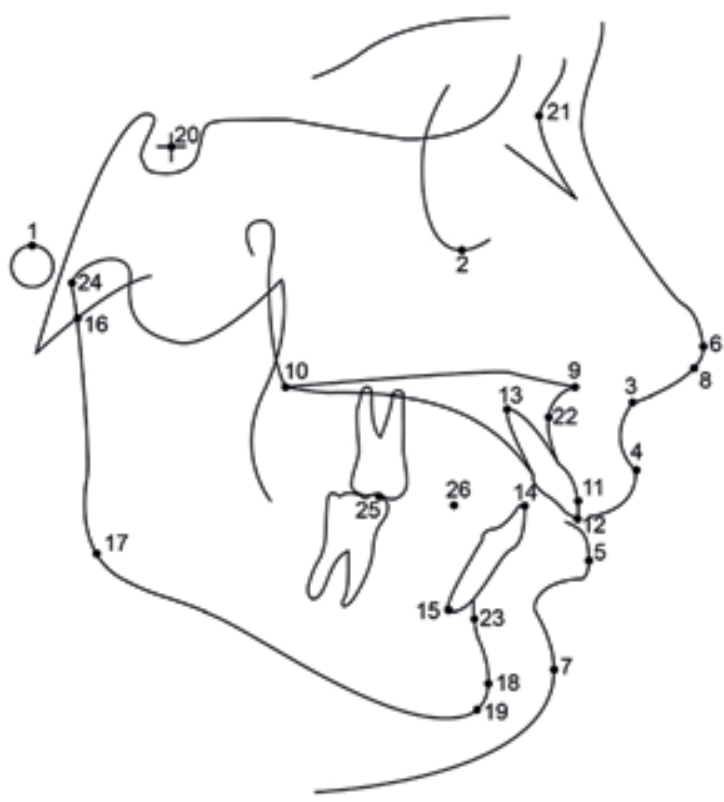

Figure 1. Lateral cephalometric landmarks. 1. Po (Porion), 2. Or (Orbital), 3. Sn (Subnasal), 4. UL (Upper Lip), 5. LL (Lower Lip), 6. Pr (Pronasale), 7. Pg' (Soft tissue Pogonion), 8. Cm (Columella), 9. ANS (Anterior Nasal Spine), 10. PNS (Posterior Nasal Spine), 11. VeU1 (Vestibular Upper Incisor), 12. InU1 (Incisal Upper Incisor), 13. ApU1 (Apical Upper Incisor), 14. InL1 (Incisal Lower Incisor), 15. ApL1 (Apical Lower Incisor), 16. Ar (Articular), 17. Go (Gonion), 18. Pg (Pogonion), 19. Gn (Gnathion), 20. $S$ (Sella), 21. $N$ (Nasion), 22. A (Subspinale), 23. B (Supramentale), 24. Co (Condylion), 25. OcM1 (First Molar Occlusal Contact), 26. OcPm (Premolars Occlusal Contact).

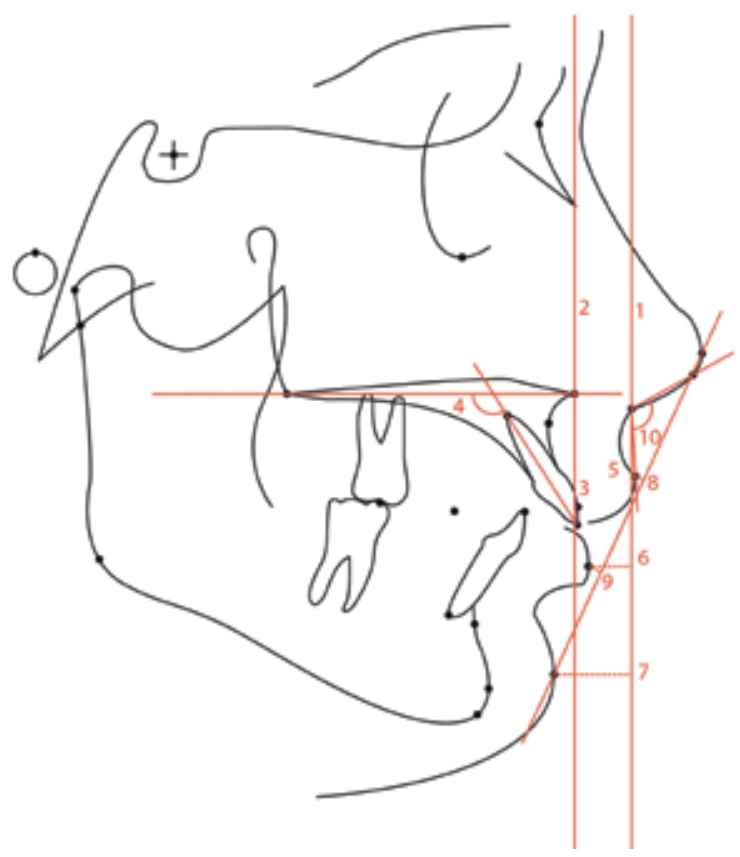

Figure 2. Soft tissue profile in lateral cephalometric. 1. TVL passing through Sn; 2. I/TVL passing through ANS; 3. I/TVL-VeU1; 4. ANS-PNS^U1; 5. UL-TVL; 6. LL-TVL; 7. Pg'-TVL; 8. UL-E-plane; 9. LL-E-plane; 10. NLA. 
among groups. Student t-test was employed for assessing any significant difference in each measurement between groups. Bonferroni correction was applied for statistically significant differences after a post-hoc analysis. The null hypothesis of this investigation stated that there was no difference among the three different groups understudy in soft tissue changes after premolars extraction, Pendulum, and MGBM treatment.

\section{Method Error}

Method of moments (MME) variance estimator was used for quantifying method error. ${ }^{22}$ Fifteen randomly selected cephalograms were retraced by a third investigator after an interval of 2 months. No significant differences between two series of cephalometric analyses were found using paired t-test. The mean error and 95\% IC between the repeated records were calculated as follows: $0.6 \mathrm{~mm}(0.5-0.8 \mathrm{~mm})$ for linear measurements and $0.7^{\circ}\left(0.6-0.9^{\circ}\right)$ for angular measurements.

\section{RESULTS}

Initial cephalometric characteristics and the pretreatment overjet of the patients in the three groups were comparable, with average overjet values of $5.52 \pm 1.02 \mathrm{~mm}$ in the U4 group, $4.76 \pm 1.32 \mathrm{~mm}$ in the PA group, and $4.63 \pm 1.36 \mathrm{~mm}$ in the MGBM group. Inferential statistical analysis of the skeletal cephalometric measurements recorded at $\mathrm{T} 1$ showed no significant differences $(p>0.05)$ in the assessed variables, indicating that the patients of the three groups were similar at the beginning of treatment. Treatments lasted between 18 and 30 months. The means and standard deviations and the p-values of the soft tissue and dento-skeletal changes between $\mathrm{T} 0$ and $\mathrm{T} 1$ within each group are summarized in Table 2, 3, and 4, while Table 5 reports the intergroup differences.

\section{Skeletal changes}

No statistically significant changes ( $>0.05)$ was detected in sagittal skeletal values between the three groups before and after treatment. SNA showed a slight decrease after treatment in all three groups, but these changes were neither statistically nor clinically significant. $\mathrm{SNB}$ and $\mathrm{SN} \wedge \mathrm{Pog}$ increased in T2-T1 changes. Mandibular length (Co-Pg) significantly increased in all three groups without any significant intergroup difference $(\mathrm{p}>0.05)$. A slight increase in vertical facial dimension $\left(\mathrm{SN}^{\wedge} \mathrm{GoGn}\right)$ in PA and MGBM groups was observed after treatment, whereas no vertical change was detected in the U4 group; however, these last modifications were not statistically relevant.

\section{Dental changes}

Before Class II treatment, the VeU1 of all three groups were protruded and positioned anteriorly the vertical line parallel to TVL and passing across ANS (//TVL-VeU1). Similar final incisor position little posterior to this reference line was found, with a greater amount of necessary retraction in the U4 group compared to PA and MGBM groups. Treatment plan resulted in an $\mathrm{VeU} 1$ retraction (//TVL-VeU1) statistically significant between U4 and PA groups $(\mathrm{p}=0.004)$ and between U4 and MGBM groups $(\mathrm{p}=0.001)$ but no significant $(\mathrm{p}>0.05)$ among PA and MGBM groups. The change of inclination of U1 relative to bispinal plane (ANS-PNS^U1) was not significantly different $(\mathrm{p}>0.05)$ in U4 group compared to MGBM group, but it increased significantly in U4 group than PA ( $\mathrm{p}=0.001)$ group and MGBM ( $\mathrm{p}=0.019)$ group. Overjet reduction was statistically relevant in U4 group than PA group $(\mathrm{p}=0.016)$ and MGBM group $(\mathrm{p}=0.05)$. Other dental values in mandibular arch changes were not statistically nor clinically relevant.

Table 2. Mean and Standard Deviation (SD) of cephalometric values and differences between T2-T1 in U4 group.

\begin{tabular}{|c|c|c|c|c|c|c|c|c|}
\hline \multicolumn{9}{|c|}{ Pre-post U4 extraction group } \\
\hline & \multicolumn{2}{|c|}{ T1 } & \multicolumn{2}{|c|}{ T2 } & \multicolumn{2}{|c|}{$\Delta \mathrm{T} 2-\mathrm{T} 1$} & \multirow{2}{*}{\multicolumn{2}{|c|}{$p$-value }} \\
\hline & Mean & SD & Mean & SD & Mean & SD & & \\
\hline \multicolumn{9}{|l|}{ Soft tissue } \\
\hline UL-TVL (mm) & 1.22 & 0.72 & 0.02 & 0.01 & -1.20 & 1.02 & 0.002 & * \\
\hline LL-TVL (mm) & -2.52 & 1.41 & -3.29 & 1.63 & -0.77 & 0.35 & 0.085 & \\
\hline UL-EPlane (mm) & -2.10 & 0.81 & -4.31 & 2.03 & -2.21 & 1.81 & 0.000 & * \\
\hline LL-EPlane (mm) & -0.66 & 0.19 & -2.39 & 0.48 & -1.73 & 1.26 & 0.001 & * \\
\hline NLA $\left(^{\circ}\right)$ & 109.43 & 2.44 & 112.02 & 2.58 & 2.58 & 0.55 & 0.149 & \\
\hline Pg'-TVL (mm) & -10.35 & 5.07 & -9.97 & 5.60 & 0.38 & 0.09 & 0.630 & \\
\hline \multicolumn{9}{|l|}{ Dental } \\
\hline //TVL-VeU1 (mm) & 2.41 & 1.18 & -0.61 & 0.37 & -3.00 & 2.94 & 0.000 & * \\
\hline ANS-PNS^U1 $\left(^{\circ}\right)$ & 113.85 & 2.77 & 109.02 & 2.48 & -4.83 & 0.51 & 0.000 & * \\
\hline $\operatorname{IMPA}\left({ }^{\circ}\right)$ & 97.17 & 2.66 & 100.80 & 2.67 & 3.63 & 0.52 & 0.004 & * \\
\hline OVJ (mm) & 5.52 & 1.02 & 2.36 & 0.86 & -3.16 & 1.07 & 0.000 & * \\
\hline OVB (mm) & 3.67 & 0.99 & 1.70 & 0.86 & -1.98 & 0.62 & 0.000 & * \\
\hline $\mathrm{U} 1 \wedge \mathrm{L} 1\left(^{\circ}\right)$ & 123.53 & 9.46 & 124.95 & 7.93 & 1.42 & 0.81 & 0.435 & \\
\hline \multicolumn{9}{|l|}{ Skeletal } \\
\hline SNA $\left({ }^{\circ}\right)$ & 80.65 & 2.58 & 78.95 & 1.51 & -1.70 & 0.24 & 0.022 & * \\
\hline $\mathrm{SNB}\left({ }^{\circ}\right)$ & 76.00 & 1.99 & 77.92 & 2.19 & 1.92 & 0.53 & 0.273 & \\
\hline $\mathrm{SN}^{\wedge} \mathrm{Pg}\left({ }^{\circ}\right)$ & 77.20 & 1.07 & 79.61 & 1.91 & 2.41 & 1.02 & 0.295 & \\
\hline Co-Pg (mm) & 106.35 & 2.98 & 110.48 & 3.34 & 4.13 & 1.93 & 0.000 & * \\
\hline $\mathrm{SN}^{\wedge} \mathrm{GoGn}\left({ }^{\circ}\right)$ & 32.52 & 2.63 & 32.62 & 3.10 & 0.10 & 0.03 & 0.822 & \\
\hline ANS-PNS^ GoGn $\left(^{\circ}\right)$ & 25.15 & 2.55 & 25.32 & 3.81 & 0.17 & 0.09 & 0.731 & \\
\hline $\mathrm{SN}^{\wedge} \mathrm{OcP}\left({ }^{\circ}\right)$ & 17.83 & 2.65 & 18.03 & 2.67 & 0.20 & 0.11 & 0.765 & \\
\hline $\mathrm{SN}^{\wedge} \mathrm{ANS}-\mathrm{PNS}\left({ }^{\circ}\right)$ & 6.95 & 2.65 & 7.23 & 2.65 & 0.28 & 0.09 & 0.138 & \\
\hline
\end{tabular}

${ }^{*} \mathrm{p}<0.05$. 
Table 3. Mean and Standard Deviation (SD) of cephalometric values and differences between T2-T1 in PA group

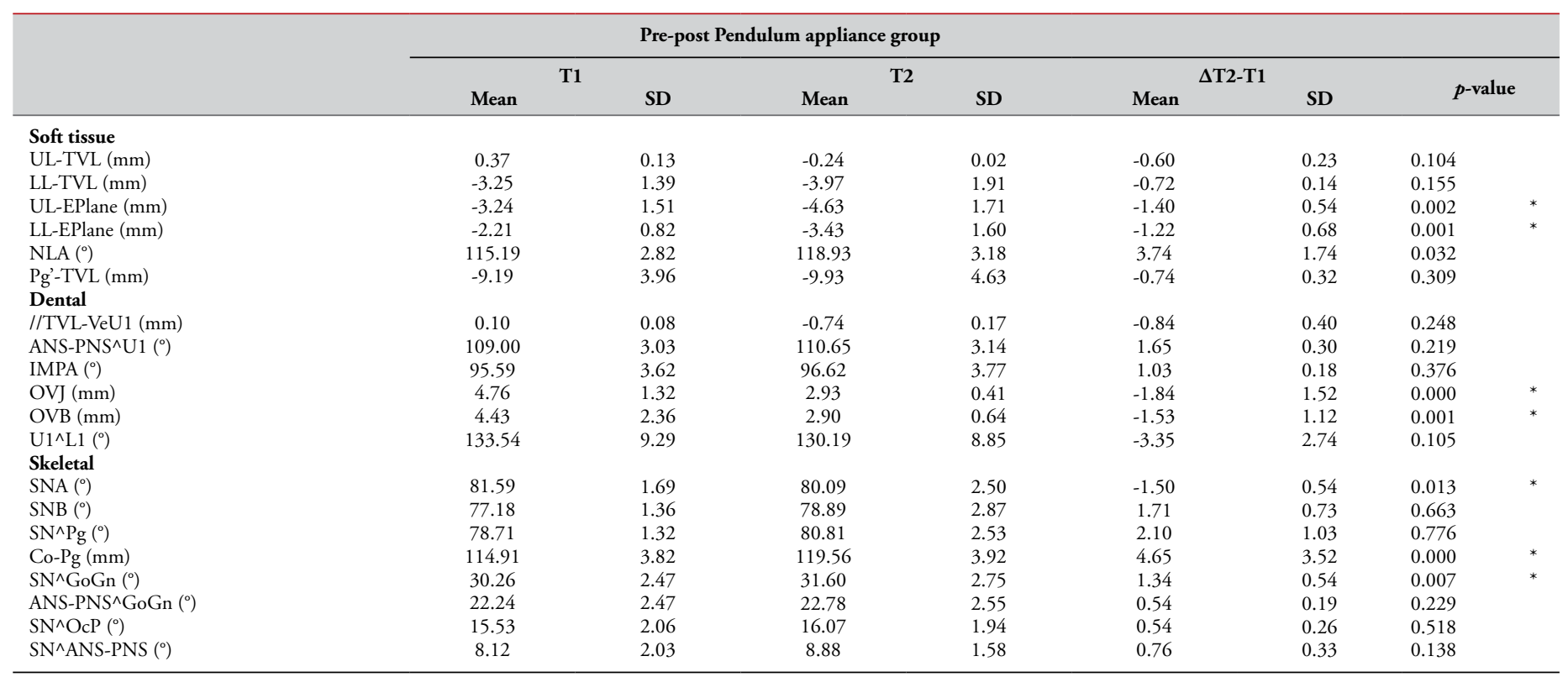

${ }^{*} \mathrm{p}<0.05$.

Table 4. Mean and Standard Deviation (SD) of cephalometric values and differences between T2-T1 in MGBS group.

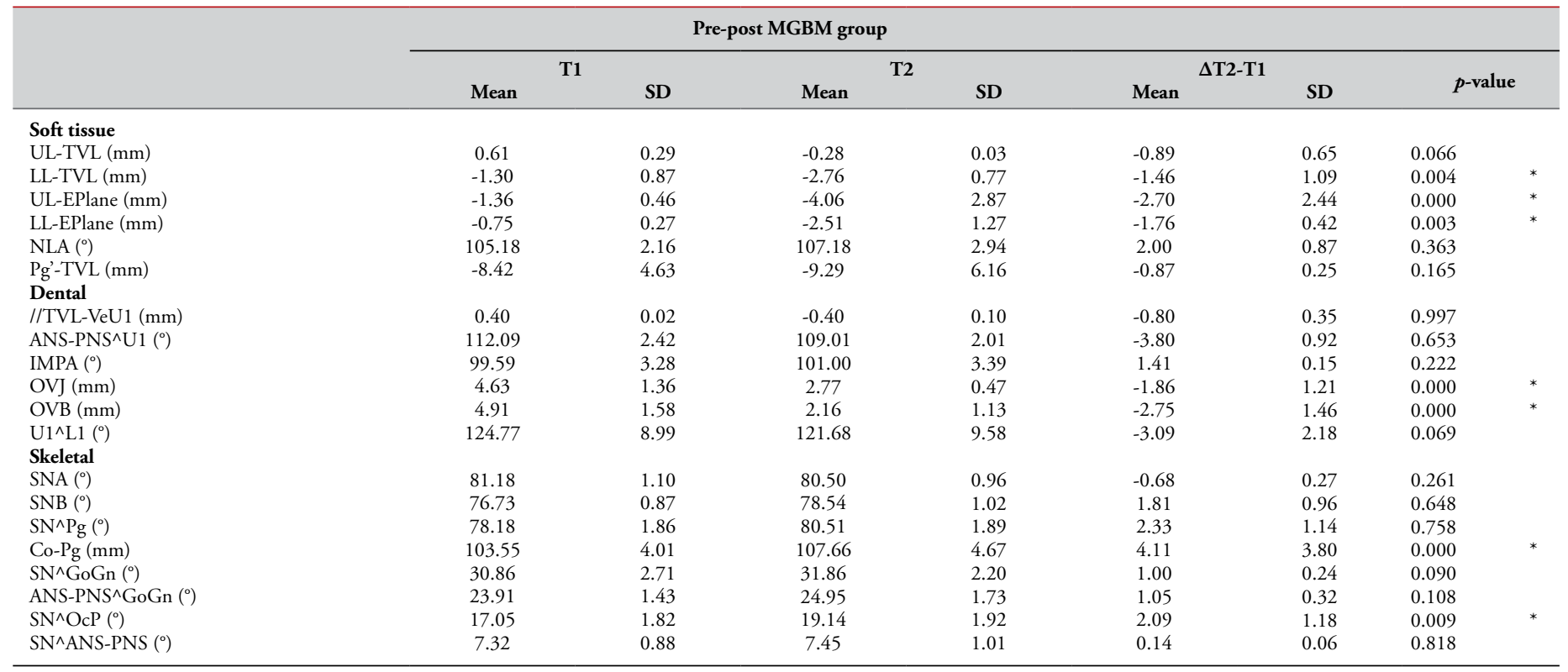

${ }^{*} \mathrm{p}<0.05$.

\section{Soft tissue and profile changes}

The inferential analysis did not show any statistically significant differences $(p>0.05)$ in soft tissue values between U4, PA, and MGBM groups. All three treatment modalities led to UL retraction when measured relative to TVL and relative to the E-plane, without any significant intergroup differences $(p>0.05)$; UL retraction was always much less than U1 retraction. No significant differences ( $p>0.05)$ were found in LL retraction regard to the TVL and E-plane. NLA mildly increased in each group without any significant intergroup differences $(p>0.05)$. Chin projection (Pg'-TVL) improved more in the U4 group than PA and MGBM groups even without statistically nor clinically significance. 
Table 5. Multiple comparisons of values differences T2-T1 time lapse in U4, PA, and MGBM groups.

\begin{tabular}{|c|c|c|c|c|c|c|}
\hline \multicolumn{7}{|c|}{ Pre-Post multiple comparison } \\
\hline & \multicolumn{2}{|c|}{ U4 } & \multicolumn{2}{|c|}{ PA } & \multicolumn{2}{|c|}{ MGBM } \\
\hline & Mean & SD & Mean & SD & Mean & SD \\
\hline \multicolumn{7}{|l|}{ Soft tissue } \\
\hline UL-TVL (mm) & -1.20 & 1.02 & -0.60 & 0.23 & -0.89 & 0.65 \\
\hline LL-TVL (mm) & -0.77 & 0.35 & -0.72 & 0.14 & -1.46 & 1.09 \\
\hline UL-EPlane (mm) & -2.21 & 1.81 & -1.40 & 0.54 & -2.70 & 2.44 \\
\hline LL-EPlane $(\mathrm{mm})$ & -1.73 & 1.26 & -1.22 & 0.68 & -1.76 & 0.42 \\
\hline NLA $\left(^{\circ}\right)$ & 2.58 & 0.55 & 3.74 & 1.74 & 2.00 & 0.87 \\
\hline Pg'-TVL (mm) & 0.38 & 0.09 & -0.74 & 0.32 & -0.87 & 0.25 \\
\hline \multicolumn{7}{|l|}{ Dental } \\
\hline //TVL-VeU1 (mm) & -3.00 & $2.94 \ddagger \dagger$ & -0.84 & $0.40 *$ & -0.80 & $0.35 *$ \\
\hline ANS-PNS^U1 $\left(^{\circ}\right)$ & -4.83 & $0.51 \ddagger$ & 1.65 & $0.30^{*} \dagger$ & -3.80 & 0.92 \\
\hline $\operatorname{IMPA}\left({ }^{\circ}\right)$ & 3.63 & 0.52 & 1.03 & 0.18 & 1.41 & 0.15 \\
\hline OVJ $(\mathrm{mm})$ & -3.16 & $1.07 \ddagger \dagger$ & -1.84 & $1.52 *$ & -1.86 & $1.21 *$ \\
\hline OVB (mm) & -1.98 & 0.62 & -1.53 & 1.12 & -2.75 & 1.46 \\
\hline $\mathrm{U} 1^{\wedge} \mathrm{L} 1\left(^{\circ}\right)$ & 1.42 & 0.81 & -3.35 & 2.74 & -3.09 & 2.18 \\
\hline \multicolumn{7}{|l|}{ Skeletal } \\
\hline SNA $\left({ }^{\circ}\right)$ & -1.70 & 0.24 & -1.50 & 0.54 & -0.68 & 0.27 \\
\hline SNB $\left({ }^{\circ}\right)$ & 1.92 & 0.53 & 1.71 & 0.73 & 1.81 & 0.96 \\
\hline $\mathrm{SN}^{\wedge} \mathrm{Pg}\left({ }^{\circ}\right)$ & 2,41 & 1.02 & 2,1 & 1.03 & 2.33 & 1.14 \\
\hline $\mathrm{Co}-\mathrm{Pg}(\mathrm{mm})$ & 4.13 & 1.93 & 4.65 & 3.52 & 4.11 & 3.80 \\
\hline $\mathrm{SN}^{\wedge}{ }^{\circ} \mathrm{GoGn}\left({ }^{\circ}\right)$ & 0.10 & 0.03 & 1.34 & 0.54 & 1.00 & 0.24 \\
\hline ANS-PNS^ GoGn $\left({ }^{\circ}\right)$ & 0.17 & 0.09 & 0.54 & 0.19 & 1.05 & 0.32 \\
\hline $\mathrm{SN}^{\wedge} \mathrm{OcP}\left(^{\circ}\right)$ & 0.20 & 0.11 & 0.54 & 0.26 & 2.09 & 1.18 \\
\hline SN^ANS-PNS $\left(^{\circ}\right)$ & 0.28 & 0.09 & 0.76 & 0.33 & 0.14 & 0.06 \\
\hline
\end{tabular}

* significant respect to U4 group; † significant respect MGBM group; $\ddagger$ significant respect PA group; $\mathrm{p}<0.05$.

\section{DISCUSSION}

The aim of the present study was to investigate orthodontic treatment for Class II patients, with an increased OVJ, performed with maxillary first premolar extractions, tooth-borne or boneborne distalizing mechanics, would have different impacts on the soft tissue outcomes, especially regard to lips projection. The statistical analysis showed that the OVJ correction of Class II malocclusion could be performed by upper first premolar extraction or upper molars distalization without any significant differences in the final soft tissue position. Basing on the obtained results, the null hypothesis was rejected.

Since skeletal structures form the foundation on which the soft tissue rest, it is understandable that the opinion has been widely held that corresponding soft tissue changes would accompany changes in skeletal structures. ${ }^{1}$ In fact, in Class II malocclusion, the protrusion and position of the UL reflected the extent of maxillary protrusion due to skeletal and dental structures. Successful orthodontic treatment is not only to establish a balanced, stable and beautiful occlusal relationship but also to achieve coordinated soft tissue profile and appearance. ${ }^{23}$

Soft tissue modification after camouflage therapy with premolar extraction in Class II patients have been previously investigated. ${ }^{16}$ Several factors may influence treatment changes on the soft tissue profile, such as mechanics, anchorage devices used, and phenotypic differences. Previous studies available in literature agree with the retraction of the UL more than LL in first upper premolar extraction protocols..$^{10,24,25}$ Moreover, NLA value also used to increase after U4 extractions; NLA is made up of both the soft tissue of the nose, which continues to grow forward, as well the soft tissue of the UL. If the UL projection is decreasing while nasal base projection remains the same, the NLA might become more obtuse as demonstrated by the results of the present study. In the U4 group, a greater amount of UL retrusion was assessed due to the necessity for greater maxillary incisor retraction, as these patients exhibited more prominent U1 with a greater OVJ and more UL prominence at T1; however in U4 group, at T1 the UL was located exactly on the TVL $(0.02 \pm 0.01 \mathrm{~mm})$, in contrast to the PA $(-0.24 \pm 0.02 \mathrm{~mm})$ and MGBM $(-0.28 \pm-0.89 \mathrm{~mm})$ groups, in which it was located slightly posteriorly. This finding is of interest if we consider that roughly $2 \mathrm{~mm}$ greater upper incisor retraction and $1.5 \mathrm{~mm}$ greater OVJ reduction was assessed in extraction treatment in contrast to the distalizing group. Therefore, the authors suppose that maxillary first premolar extractions do not necessarily lead to more lip retraction than any distalizing mechanics, if the therapeutic indication is correct.

The distalization of maxillary molars is the frequently used nonextraction treatment in Class II malocclusion to establish a Class I molar and canine relationships. Pendulum device permits the molar class correction accomplished through dentoalveolar changes rather than maxillary growth restriction. Previous study, comparing Pendulum and premolar extraction treatments, is available about soft tissue reaction to PA to compare our data with ${ }^{26}$ it has been shown that PA produces significant differences with regard to lips position which were greater in PA group compared to U4 group, in contrast with the results of the present study. The main limitation with this appliance is anchorage loss; the forces directed to anterior teeth and the movements exercised by the activators caused movement of anchoring teeth with anchorage loss. ${ }^{27}$ This could be the reason of an increase values of incisor's inclination (ANS-PNS^U1) in PA group than U4 and MGBM groups. Due to that, PA is more appropriated in Class II patients with reduced U1 buccal inclination. 
To prevent anchorage loss, characteristic of dental-bonded devices, skeletal anchorage has been introduced in orthodontics. With the coming of dental implants, mini plates, and miniscrews as anchorage units, it has now become possible to obtain absolute anchorage for teeth movements. A systematic review about molar distalization with skeletal-supported appliances in Class II malocclusion has been recently published, although it, unfortunately, did not analyze soft tissue modifications. ${ }^{28}$ The main advantage of the use of skeletal anchorage compared to dental-borne appliances is the prevention of the flaring of maxillary incisors caused by anchorage loss. The results obtained in the present study showed that there are not statistically significative differences in soft tissue changes between MGBM system and PA; therefore, MGBM ensures class molar correction without the drawbacks of PA like incisor labial tipping.

Regardless of the technique used, it is essential to consider the accordance between diagnosis and treatment as a fundamental part of orthodontic practice. The test subjects have been selected with specific criteria, such as a moderate Class II with at least dental end-to-end Class II molar relationships and consequently reduced overjet, that could be virtually and successfully performed with only an orthodontic treatment. The results of the present study are to be considered reliable, respecting the possibility of dealing with these cases only with an orthodontic approach. Therefore, cases with similar selection criteria could be approached with similar final results in regard of soft tissue. In the remaining cases of severe dental and skeletal discrepancies of Class II malocclusion, a multidisciplinary orthodonticorthognatic treatment is required, ${ }^{8}$ in order to restore not only dental but also skeletal relationships with essential involvement of face profile; in these cases, a surgical visual treatment objective is required to preview a face rendering.

Lip structure seems to have an influence on lip response to incisor retraction. High correlation between soft tissue change and osseous change after incisor retraction, and different soft tissue responses have been explained by soft tissue thickness and lip strain..$^{25,29}$ Consequently, a greater retraction of the upper and lower incisors gives more opportunity for lips to move posteriorly. Lip response, as a proportion of incisor retraction, decreased as the amount of incisor retraction increased; so, the ratio of maxillary incisor retraction to posterior movement of the UL cannot be 1:1.1 In fact UL does not show steady and predictable response to retraction of upper incisors, as confirmed in the present study. This seems to indicate that the lips have some inherent support that influences the soft tissue profile.

When assessing therapeutic changes in the lip profile, it is also important to discuss growth-related changes. ${ }^{23,30}$ Lip's tissue continues to change until adulthood. The lips of adolescents become relatively more retrusive over time, substantially longer, and thicker, while the regions of nose and chin exhibit anterior growth changes in adolescents. For these reasons, the results of the present study are clinically relevant in growing Class II patients, but they might not be significant in adulthood.

Class II correction and normalization of the increased OVJ was mainly achieved through a combination of dentoalveolar maxillary incisor retraction and mandibular growth, especially in patients with an incomplete pubertal spurt. Mandibular growth could be the reason to explain the increase of $\mathrm{SNB}$ and $\mathrm{SN} \wedge \mathrm{Pog}$ cephalometric angles during treatment observed in the present study; the same hypothesis could explain the stable chin projection (Pg-TVL) values at the end of orthodontic treatment while CoPg distance increased. Chin is considered to be one of the most important index of soft tissue profile in the evaluation standard of Class II patients due to a frequently mandibular retrusion. Moreover, with the bite opening that occurred in distalizing methods, it is possible to see a downward and backward rotation of the chin, getting worse retracted chin profile. ${ }^{24}$ In patients before a pubertal growth spurt, the remaining minimal mandibular growth is sufficient to overcome the mild increase in face height, without reducing the chin projection. The chin projection due to mandibular growth can also explain the retrusion of LL while IMPA value increases in all the three treatments group.

U1 retraction provoked a decrease of both UL and LL projection relative to the TVL and to the E-plane and a more obtuse NLA, which reflects a straighter post-treatment profile, ${ }^{31}$ independently of treatment approaches. However, the E-plane is not a reliable reference plane owing to the simultaneous changes in the Pg' and Pr point. ${ }^{26}$ For this reason, it may be advisable to utilize the TVL for analysis of the soft tissue profile, as it is less influenced by nasal and chin growth. ${ }^{32}$ Nevertheless, in the present investigation no statistically significant differences could be evaluated depending on the choice of the reference planes.

No significant clinical differences between the final upper and lower lip projection between conventional and skeletal distalization therapy and maxillary premolar extraction treatments for Class II malocclusion patients with increased OVJ could be detected. With all three treatment approaches a mild retraction of the upper and lower lips due to the necessary maxillary dentoalveolar changes and to some mandibular anterior growth have to be expected, with little predictability of the individual response. None of the corrective mechanics proved to be superior to the other. The degree to which profile changes occur is likely to be variable and does not seem to depend primarily on the applied mechanics but on the final angulations of the upper and lower incisors, the pretreatment lip thickness, and the vertical and anteroposterior facial patterns. ${ }^{29}$

\section{CONCLUSIONS}

In absence of a skeletal discrepancy and mandibular crowding, the overjet correction can be performed without any significant differences in the soft tissue at the end of Class II orthodontic treatment. Based on the result of the present study it can be concluded that neither first premolar extractions, molar distalization by Pendulum device, or molar distalization by MGBM system do not affect face profile.

\section{COMPETING INTERESTS}

The authors declare that they have no competing interests. No funding was received. 


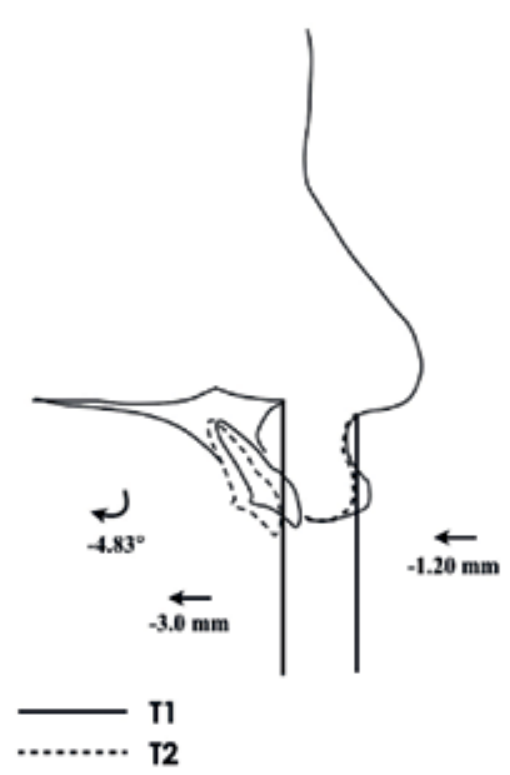

Figure 3. Maxillary superimposition for U4 group showing dental and upper lip changes.

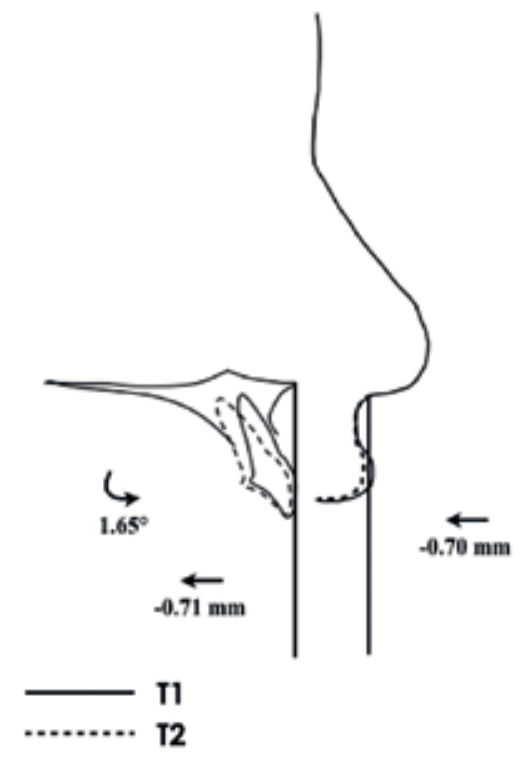

Figure 4. Maxillary superimposition for $P A$ group showing dental and upper lip changes.

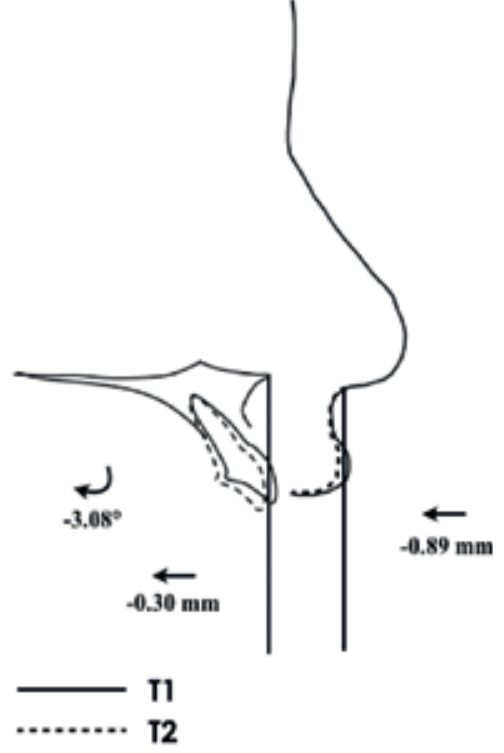

Figure 5. Maxillary superimposition for $M G B M$ group showing dental and upper lip changes.

\section{REFERENCES}

1. Roos N. Soft-tissue profile changes in class II treatment. Am J Orthod. 1977;72:165-75.

2. Hodges A, Rossouw PE, Campbell PM, Boley JC, Alexander RA, Buschang PH. Prediction of lip response to four first premolar extractions in white female adolescents and adults. Angle Orthod. 2009;79:413-21.

3. Flores-Mir C, Major MP, Major PW. Soft tissue changes with fixed functional appliances in Class II division 1. Angle Orthod. 2006;76:712-20.

4. D’Antò V, Bucci R, Franchi L, Rongo R, Michelotti A, Martina R. Class II functional orthopaedic treatment: a systematic review of systematic reviews. J Oral Rehabil. 2015;42:624-42.

5. Sambataro S, Fastuca R, Oppermann NJ, Lorusso P, Bacetti T, Franchi L, Caprioglio A. Cephalometric changes in growing patients with increased vertical dimension treated with cervical headgear. J Orofac Orthop. 2017;78:312-20.

6. Ghosh J, Nanda RS. Evaluation of an intraoral maxillary molar distalization technique. Am J Orthod Dentofacial Orthop. 1996;110:639-46.

7. Janson G, Brambilla Ada C, Henriques JF, de Freitas MR, Neves LS. Class II treatment success rate in 2- and 4-premolar extraction protocols. Am J Orthod Dentofacial Orthop. 2004;125:472-9.

8. Pancherz H, Ruf S, Erbe C, Hansen K. The mechanism of Class II correction in surgical orthodontic treatment of adult Class II, division 1 malocclusions. Angle Orthod. 2004;74:800-9.

9. Pisani L, Bonaccorso L, Fastuca R, Spena R, Lombardo L, Caprioglio A. Systematic review for orthodontic and orthopedic treatments for anterior open bite in the mixed dentition. Prog Orthod. 2016;17:28.

10. Janson G, Fuziy A, de Freitas MR, Castanha Henriques JF, de Almeida RR. Soft-tissue treatment changes in Class II Division 1 malocclusion with and without extraction of maxillary premolars. Am J Orthod Dentofacial Orthop. 2007;132:729.e1-e8.

11. Quaglio CL, de Freitas KM, de Freitas MR, Janson G, Henriques JF. Stability and relapse of maxillary anterior crowding treatment in class I and class II Division 1 malocclusions. Am J Orthod Dentofacial Orthop. 2011;139:768-74.

12. Hilgers JJ. The pendulum appliance for Class II non-compliance therapy. J Clin Orthod. 1992;26:706-14.

13. Bussick TJ, McNamara JA Jr. Dentoalveolar and skeletal changes associated with the pendulum appliance. Am J Orthod Dentofacial Orthop. 2000;117:333-43.

14. Maino BG, Gianelly AA, Bednar J, Mura P, Maino G. MGBM system: new protocol for Class II non extraction treatment without cooperation. Prog Orthod. 2007;8:130-43.

15. Mariani L, Maino G, Caprioglio A. Skeletal versus conventional intraoral anchorage for the treatment of class II malocclusion: dentoalveolar and skeletal effects. Prog Orthod. 2014;15:43.

16. Janson G, Mendes LM, Junqueira CH, Garib DG. Soft-tissue changes in Class II malocclusion patients treated with extractions: a systematic review. Eur J Orthod. 2016;38:631-7.
17. de Almeida-Pedrin RR, Henriques JF, de Almeida RR, de Almeida MR, McNamara JA Jr. Effects of the pendulum appliance, cervical headgear, and 2 premolar extractions followed by fixed appliances in patients with Class II malocclusion. Am J Orthod Dentofacial Orthop. 2009;136:833-42.

18. Pinzan-Vercelino CR, Janson G, Pinzan A, de Almeida RR, de Freitas MR, de Freitas KM. Comparative efficiency of Class II malocclusion treatment with the pendulum appliance or two maxillary premolar extractions and edgewise appliances [corrected]. Eur J Orthod. 2009;31:333-40.

19. Byloff FK, Darendeliler MA. Distal molar movement using the pendulum appliance. Part 1: Clinical and radiological evaluation. Angle Orthod. 1997;67:249-60.

20. Caprioglio A, Fontana M, Longoni E, Cozzani M. Long-term evaluation of the molar movements following Pendulum and fixed appliances. Angle Orthod. 2013;83:447-54.

21. Maino G, Mariani L, Bozzo I, Maino G, Caprioglio A. Maxillary molar distalization with MGBM-system in class II malocclusion. J Orthod Sci. 2013;2:101-8.

22. Springate SD. The effect of sample size and bias on the reliability of estimates of error: a comparative study of Dahlberg's formula. Eur J Orthod. 2012;34:158-63.

23. Sarver DM. Interactions of hard tissues, soft tissues, and growth over time, and their impact on orthodontic diagnosis and treatment planning. Am J Orthod Dentofacial Orthop. 2015;148:380-6.

24. Scott Conley R, Jernigan C. Soft tissue changes after upper premolar extraction in Class II camouflage therapy. Angle Orthod. 2006;76:59-65.

25. Kim K, Choi SH, Choi EH, Choi YJ, Hwang CJ, Cha JY. Unpredictability of soft tissue changes after camouflage treatment of Class II division 1 malocclusion with maximum anterior retraction using miniscrews. Angle Orthod. 2017;87:230-8.

26. Atik E, Akarsu-Guven B, Kocadereli I. Soft tissue effects of three different Class II/1camouflage treatment strategies. J Orofac Orthop. 2017;78:153-65.

27. Fontana M, Cozzani M, Caprioglio A. Non-compliance maxillary molar distalizing appliances: an overview of the last decade. Prog Orthod. 2012;13:173-84.

28. Mohamed RN, Basha S, Al-Thomali Y. Maxillary molar distalization with miniscrewsupported appliances in Class II malocclusion: A systematic review. Angle Orthod. 2018;88:494-502.

29. Lee YJ, Park JT, Cha JY. Perioral soft tissue evaluation of skeletal Class II Division 1: A lateral cephalometric study. Am J Orthod Dentofacial Orthop. 2015;148:405-13.

30. Maetevorakul S, Viteporn S. Factors influencing soft tissue profile changes following orthodontic treatment in patients with Class II Division 1 malocclusion. Prog Orthod. 2016;17:13.

31. James RD. A comparative study of facial profiles in extraction and nonextraction treatment. Am J Orthod Dentofacial Orthop. 1998;114:265-76.

32. Bishara SE, Jacobsen JR, Hession TJ, Treder JE. Soft tissue profile changes from 5 to 45 years of age. Am J Orthod Dentofacial Orthop. 1998;114:698-706. 